\title{
Transtorno de Personalidade Borderline, tentativas de suicídio e desempenho cognitivo
}

\author{
Borderline Personality Disorder, suicide attempts and cognitive performance
}

Edilson Pastore ${ }^{[a]}$, Carolina Saraiva de Macedo Lisboa ${ }^{[b]}$

\footnotetext{
${ }^{[a]}$ Mestre em Psicologia Clínica pela Universidade do Vale do Rio dos Sinos (Unisinos), São Leopoldo, RS - Brasil. Coordenador e supervisor do Departamento de Ensino e Pesquisa da Clínica Pinel, e-mail:

edilson.pastore@clinicapinel.com.br

${ }^{[b]}$ Doutora em Psicologia, professora da Pontifícia Universidade Católica do Rio Grande do Sul (PUCRS), Porto Alegre, RS - Brasil, e-mail: carolina.lisboa@pucrs.br
}

Recebido: $22 / 12 / 2012$

Received: $12 / 22 / 2012$

Aprovado: 02/04/2013

Approved: 04/02/2013

\section{Resumo}

Introdução: 0 presente artigo apresenta uma revisão crítica e assistemática da literatura sobre o Transtorno de Personalidade Borderline (TPB), suicídio e funções cognitivas. Materiais e métodos: As bases de dados pesquisadas foram os portais Capes, ScienceDirect, PsycINFO, Cochrane, BVS-PSI, Bireme e Medline, com os seguintes descritores: "Borderline Personality Disorder", “Cognition” e "Suicidal Behavior". Desenvolvimento: Em um primeiro momento, foram abordadas as características principais do transtorno, sintomas iniciais e suas principais manifestações sintomatológicas. Posteriormente, foram enfocados os aspectos cognitivos de pacientes com TPB, abordando especialmente as funções executivas, como a tomada de decisões. Nesse sentido, foi também discutida a avaliação da impulsividade e o risco de suicídio em pacientes com TPB. Estudos atuais revisados apontam que déficits em funções neuropsicológicas, especialmente nas funções executivas, podem ser importantes fatores de risco para tentativas e também para a efetivação do suicídio em pessoas com esse diagnóstico. Considerações finais: A importância do estudo da cognição, da impulsividade e dos níveis de ideação suicida nos pacientes com TPB é fundamental para auxiliar os profissionais de saúde que trabalham com essa população a identificar e prevenir o suicídio nesses pacientes e também para se aprimorarem estratégias de tratamentos existentes. É de fundamental importância aprofundar o conhecimento acerca do TPB a partir da realização de novos estudos sobre o tema, especialmente na América Latina, onde as publicações específicas ainda são escassas.

Palavras-chave: Transtorno de Personalidade Borderline. Cognição. Suicídio.

\section{Abstract}

Introduction: This article presents a critical and unsystematic literature review on Borderline Personality Disorder (BPD), suicide and cognitive functions. Material and methods: The databases searched were: Capes, ScienceDirect, PsycINFO, Cochrane, BVS-PSI, Bireme and Medline, with the following keywords: "Borderline Personality Disorder", "Cognition" and "Suicidal Behavior". Development: At first, the main characteristics of the disorder, the early onset of symptoms and its main manifestations were addressed. Later, the cognitive aspects of patients with BPD were focused, especially addressing the executive functions such as decision making. Thus, it was also discussed the assessment of impulsivity and suicide risk in patients with BPD. Current studies 
included in this review suggest that deficits in neuropsychological functions, especially in executive functions, may be important risk factors for suicide and suicide attempts in people with this diagnosis. Final thoughts: The importance of the study of cognition, impulsivity and levels of suicidal ideation in patients with BPD is essential to help health professionals who work with this population to identify and prevent suicide in these patients and also to improve the existing treatment strategies. It is extremely important to deepen the knowledge on BPD carrying out new studies about this disorder, especially in Latin America, where specific publications are still scarce.

Keywords: Borderline Personality Disorder. Cognition. Suicide.

\section{Introdução}

Os transtornos de personalidade vêm ocupando cada vez mais espaço na literatura científica, sendo objeto tanto de revisões teóricas quanto de estudos empíricos (Brooke \& Horn, 2010; Brodsky, Malone, Ellis, Dulit, \& Mann, 2006; Paris, 2005). Esse movimento se deve ao avanço das ciências humanas, biológicas e sociais no que tange ao maior aprofundamento nesses complexos fenômenos psíquicos. Dentre os transtornos de personalidade, pode-se afirmar que há um tipo de transtorno que ocupa um lugar de importante destaque devido a sua complexidade, heterogeneidade e dificuldade de tratamento, denominado Transtorno de Personalidade Borderline (American Psychiatric Association [APA], 2002).

0 TPB tem despertado curiosidade e necessidade de maior compreensão em função da riqueza das manifestações clínicas inerentes e ao grau de complexidade dos casos (Joyce, Light, Rowe, Cloninger, \& Kennedy, 2010; Freeman \& Beck, 2000). Ao mesmo tempo em que há maior interesse nesse transtorno, observado pelo crescente número de estudos, evidencia-se que os profissionais que trabalham com essa população clínica evitam, muitas vezes, atender a esses pacientes, pois se sentem impotentes, desmotivados e desgastados em função das inúmeras dificuldades que envolvem o tratamento de portadores desse transtorno (Anestis, Selby, \& Joiner, 2007). Essas dificuldades referem-se a aspectos centrais que constituem o TPB, tais como a instabilidade afetiva, a impulsividade e a agressividade (Nigg, Lohr, Western, Gold, \& Silk, 1992), e ficam ainda mais acentuadas quando esses pacientes apresentam tentativas de suicídio associadas (Romaro, 2002). A partir disso, neste artigo apresenta-se uma revisão crítica e assistemática da literatura sobre o TPB, os aspectos cognitivos implicados nessa patologia, abordando relações interdependentes desses aspectos com o risco de suicídio apresentado por essa população.

\section{Desenvolvimento}

\section{Transtornos de personalidade}

Os transtornos de personalidade são definidos por uma série de critérios, sendo que o principal é a identificação de um padrão persistente de vivências íntimas e de comportamentos que se desviam de forma acentuada das expectativas da cultura do indivíduo. De acordo com os critérios da American Psychiatric Association (APA, 2002), o transtorno de personalidade consiste em um padrão global, rígido e inflexível com duração de mais de dois anos a partir do final da adolescência ou dos primeiros anos da vida adulta, e que afeta pelo menos duas das seguintes áreas: 1) afeto, 2) cognição, 3) relacionamentos interpessoais, 4) controle dos impulsos. Há também outros indicadores que podem auxiliar a caracterizar se uma pessoa é portadora de um transtorno de personalidade como, por exemplo, a forma de perceber e de interpretar a si e aos outros, assim como os acontecimentos a sua volta (Brodsky et al., 2006; Gabbard, 1998). Observa-se, ainda, que um indivíduo com transtorno de personalidade tem maior probabilidade de recusar e resistir ao tratamento e negar seus problemas, se comparado com indivíduos com outras patologias (Kaplan \& Sadok, 2008).

Segundo a classificação da APA (2002), os transtornos de personalidade são divididos em categorias:

- Cluster A: paranoide, esquizoide e esquizotípico. 
- Cluster B: antissocial, histriônico, narcisista e borderline.

- Cluster C: esquiva, dependente, obsessivo compulsivo e sem outra especificação.

\section{Transtorno de Personalidade Borderline}

O TPB é um distúrbio estrutural da personalidade que se caracteriza por um padrão invasivo e conturbado de relacionamentos interpessoais, bem como alterações na forma de perceber a si mesmo e em relação aos afetos (Malloney, Degenhardt, Darke, \& Nelson, 2009; Sollof et al., 2012; Kernberg, 1998). Observa-se também em pacientes com esse diagnóstico um acentuado grau de impulsividade, sendo que as primeiras manifestações tendem a ocorrer no final da adolescência ou no início da idade adulta, permanecendo presentes em vários contextos da vida do paciente, com tendência à remissão parcial da sintomatologia a partir da quarta década de vida (Lieb, Zanarini, Schmahl, Linehan, \& Bohus, 2004). Segundo Kaplan e Sadok (2008), esta condição diagnóstica era definida por termos como esquizofrenia ambulatória, personalidade "como se", esquizofrenia pseudoneurótica, transtorno psicótico de caráter.

Para O'Donohue e Cucciare (2007), o TPB inclui um conjunto de fatores que explicam sua etiologia, sendo que os principais são: a biologia/genética, experiências de separação e perda, abuso infantil e ambiente familiar conturbado e caótico. A literatura aponta a separação precoce das figuras parentais e as perdas na infância como componentes importantes na determinação do TPB (Akiskal et al., 1985); sendo que de 20 a $40 \%$ dos pacientes que possuem diagnóstico de TPB experimentaram separação traumática de pelo menos um dos pais (Gunderson \& Sabo, 1993).

Outra questão importante sobre a etiologia do TPB refere-se a casos de abuso sexual na infância, sendo que Hermann, Perry e Van der Kolk (1989) identificaram em seu estudo que $81 \%$ dos pacientes com TPB possuíam história de abuso físico e/ ou sexual na infância. Da mesma forma, Zelkovitz, Paris, Guzder e Feldman (2001) observaram que pessoas que haviam sofrido abuso sexual apresentavam quatro vezes mais chance de desenvolver o TPB. O abuso sexual e a concomitante severidade do TPB no futuro estariam mais ligados a fatores como a idade em que ele ocorreu, a frequência em que acontecia e a gravidade/intensidade existente e percebida pelos indivíduos (McLean \& Gallop, 2003; Zanarini, Frankenburg, \& Vujanovic, 2002).

Ambiente familiar caótico e conturbado constitui também forte preditor para o desenvolvimento do TPB, pois, segundo Johnson et al. (2001), relacionamentos com vinculações problemáticas, abuso emocional e negligência por parte dos cuidadores são fatores de risco para o desenvolvimento do TPB, tanto quanto o abuso sexual, que é um dos aspectos mais abordados e apontados na literatura sobre o tema (Zelkovitz et al., 2001). A vinculação insegura a figuras parentais e ambientes familiares conturbados e emocionalmente instáveis e negligentes também podem explicar o TPB tanto quanto os demais fatores apontados pela literatura (Salzman et al., 1993). Outro fator para tentar explicar a etiologia do TPB diz respeito à questão da vinculação da criança com seus cuidadores e o desenvolvimento de apego inseguro (Bowlby, 1969/1973). Pesquisas relacionando o TPB com o apego inseguro são frequentes na literatura e apontam que experiências com cuidadores inseguros, imprevisíveis, assustadores ou abusivos predisporiam a criança a um tipo de apego que poderia ser um preditor para o desenvolvimento do TPB no futuro (Levy et al., 2006; Lyons-Ruth, Jacobvitz, Cassidy, \& Shaver, 1999).

Atualmente o DSM IV-TR (APA, 2002) denomina esse transtorno como Transtorno de Personalidade Borderline e o CID-10 (Organização Mundial da Saúde [OMS], 1994) o classifica como Transtorno de Personalidade Emocionalmente Instável, sendo ambas as categorias diagnósticas clinicamente equivalentes. A prevalência do TPB é de aproximadamente $2 \%$ a $7 \%$ da população, sendo três vezes mais comum em mulheres do que em homens (Links, Heisel, \& Garland, 2003). Encontra-se, ainda, entre os principais critérios para definir o TPB, a identificação de esforços feitos pelo paciente para evitar abandonos, reais ou imaginários, significativa instabilidade nos relacionamentos, alternância de extremos de idealização e desvalorização, acentuada perturbação na identidade e na autoimagem, desregulação afetiva, assim como impulsividade em diversas áreas de sua vida (Kernberg, 1998). Convém salientar que devem constar pelo menos dois indicadores das seguintes alterações: sexualidade, abuso de substâncias, direção imprudente ou comer compulsivo para que o diagnóstico de TPB possa ser conclusivo 
(APA, 2002). Sentimentos crônicos de vazio, raiva inadequada, dificuldades importantes nos relacionamentos, incluindo lutas corporais, também estão, muitas vezes, presentes nesses pacientes (Gabbard \& Wilkinson, 1994).

Um dos critérios mais importantes e também mais preocupantes do TPB diz respeito às ameaças e tentativas de suicídio que acometem esses pacientes (Linehan, 2010; Brodsky et. al., 2006). Muitas dessas tentativas constituem-se em uma forma de chamar atenção para o sofrimento emocional que vivenciam e não chegam a constituir risco real para que cheguem a óbito. Ainda assim, toda tentativa de suicídio requer atenção cautelosa e cuidados intensivos. No caso desses pacientes, essa atenção deve ser aumentada devido ao risco potencial para automutilações denominadas também como comportamentos parasuicidas e, evidentemente, à possibilidade de ocorrer efetivamente o suicídio (Linehan, 2010; Paris, 2005; Koenigsberg, 2002). De acordo com Links et al. (2003), resultados de autópsia psicológica de mortes por suicídio estimam que entre $9 \%$ e $33 \%$ dos pacientes que se suicidaram preenchiam critérios diagnósticos para TPB. Ainda segundo os autores, compreender os fatores que fazem o risco de suicídio aumentar, assim como o comportamento suicida e a própria ideação suicida, são cruciais para o entendimento do TPB.

Entende-se por suicídio um ato deliberado do indivíduo contra si mesmo, com a finalidade de morrer (OMS, 2001; Brodsky et al., 2006). Os suicídios consumados em pacientes que apresentam TPB giram em torno de $10 \%$, e as tentativas de suicídio entre essa população variam de $37 \%$ a 73 \% (Black, Blum, Pfohl, \& Hale 2004). Segundo Paris (2005), um em cada dez pacientes com diagnóstico de ТРB se suicida, igualando-se o índice aos de patologias com alta letalidade como a esquizofrenia e a depressão maior. Esses dados levam a questionar uma ideia preconcebida e estigmatizada de que pacientes com TPB tentam suicídio apenas para manipular ou chamar a atenção (Linehan, 2010).

Em um estudo realizado por Brooke e Horn (2010), pacientes com TPB que apresentavam automutilações e tentativas de suicídio também apresentaram baixa autoestima e visão negativa de si. Observou-se que consequências psicológicas de abusos físicos e emocionais foram determinantes ou desencadeantes para as tentativas de suicídio na amostra estudada. Ainda segundo esse estudo, a impossibilidade de tolerar a solidão e o intenso vazio mostrou-se associada ao aumento significativo da ansiedade, o que pode explicar que o paciente queira atentar contra a própria vida.

Assim, de acordo com uma série de autores (Arza et al., 2009; Domes et al., 2006; Le Gris \& Van Reekum, 2006; Monarch, Saykin, \& Flashman, 2004; Soler et al., 2009), a relação entre TPB e o comportamento suicida pode ser mediada por aspectos cognitivos, especialmente envolvendo dificuldades relacionadas a tomada de decisões, memória de trabalho, capacidade para obter juízo crítico e formulação de insight (Le Gris \& Van Reekum, 2006). Esses aspectos podem auxiliar na explicação de uma série de variáveis ainda obscuras na literatura e servir de subsídios para melhores estratégias de tratamento.

\section{Aspectos cognitivos}

Achados neuropsicológicos em indivíduos com TPB demonstram que há falhas importantes nessa esfera, ressaltando que mais estudos devem ser realizados para aprofundar a investigação a respeito desse tema (Le Gris \& Van Reekum, 2006; O'Connor $\&$ Noyce, 2008). De acordo com Monarch et al. (2004), apesar das evidências acumuladas sobre possíveis prejuízos em funções neuropsicológicas de pacientes com TPB, o mesmo ainda não configura um transtorno que apresente características cognitivas específicas. Assim, de acordo com o autor, estudos que objetivem conhecer mais profundamente o TPB e suas particularidades da cognição devem ser conduzidos.

Pesquisas sobre a etiologia do TPB afirmam que variáveis como genética e traumas infantis, tais quais a negligência, abuso sexual e emocional, além de outros eventos estressores, podem ser causas para esse transtorno e seriam responsáveis por falhas cognitivas (Mann, 2003). Por outro lado, autores como Le Gris e Van Reekum (2006) e Ruocco (2005) afirmam que pacientes com TPB apresentam comprometimento de domínios cognitivos específicos ligados a funções como atenção, flexibilidade cognitiva, memória de trabalho, capacidade de planejamento e memória visoespacial. A partir desses resultados, verifica-se a necessidade de uma compreensão acerca do funcionamento cognitivo de pacientes com TPB, pois se trata de uma doença complexa, na qual o comportamento dos pacientes 
é provavelmente dependente de múltiplos fatores como os neurobiológicos, intelectuais, psicológicos e/ou ocorrência de situações estressoras ao longo do desenvolvimento (Brezo, Paris, \& Turecki, 2006). Portanto, investigações da esfera cognitiva junto a pacientes com TPB podem contribuir para a compreensão das possíveis dificuldades psicológicas associadas a esse transtorno, aprimorando as suas formas de tratamento existentes.

Avançando dentro da complexa heterogeneidade do TPB, estudos apontam que há um comprometimento cognitivo maior nos pacientes suicidas em relação à população geral e também comparados a indivíduos com TPB sem ideação suicida (Le Gris \& Van Reekum, 2006). Tais indicadores incluem dificuldade na resolução de problemas, déficits de memória e na atenção, estilo cognitivo negativo, impulsividade e falta de capacidade de planejamento (O’Connor \& Noyce, 2008).

Estudos de neuroimagem cerebral mostram evidências de interrupção nos circuitos neurais em áreas do cérebro responsáveis pelos mecanismos intelectuais, agressivos e de regulação afetiva em pacientes com TPB (New, Goodman, Triebwasser, \& Siever, 2008). Corroborando esses resultados, Bazanis et al. (2002) afirmam que pacientes com TPB apresentam déficits na capacidade de tomada de decisões e de planejamento. Além disso, apresentam outras dificuldades intelectuais, respondendo de forma impulsiva a determinados estímulos, realizando interpretações distorcidas de eventos cotidianos, bem como apresentando ações equivocadas e prematuras. Observa-se, ainda, que esses indivíduos suicidas sofrem de rigidez cognitiva, o que, possivelmente, os leva a acreditar que o suicídio é a sua única opção (Le Gris \& Van Reekum, 2006).

Segundo Gvion e Apter (2011), a presença de deficiências cognitivas tem sido relacionada a disfunções nas áreas cerebrais responsáveis pelas funções executivas. Essas funções podem interagir com aspectos emocionais, alterando a capacidade que o indivíduo tem de pensar e controlar seus impulsos comportamentais, podendo gerar uma maior propensão para comportamento suicida. Polleti (2009) também aponta para disfunções cognitivas em funções executivas, especialmente na memória autobiográfica e cognição social, o que poderia tornar os pacientes com TPB mais propensos a cometer falhas no processo de avaliação e julgamento. Esse autor também aponta que o início dos danos cognitivos em pacientes com TPB pode ser observado ainda na infância e na adolescência.

Em estudo comparando os prejuízos cognitivos de 40 pacientes com TPB e 40 controles saudáveis, O'Leary, Brouwers, Gardner e Cowdry (1991) constataram que há sérios prejuízos nos pacientes com TPB. Tais déficits encontravam-se em áreas relacionadas a funções executivas que não estavam relacionadas a problemas motores, abuso de álcool, depressão maior ou outros fatores não devidos ao TPB. Assim, o autor aponta para a existência de déficits específicos em domínios cognitivos de pacientes com TPB.

Em outro contexto de estudo, a fim de explanar mais sobre as diferenças entre pacientes suicidas e não suicidas, uma investigação neuropsicológica em pacientes com diagnóstico de depressão evidenciou que tentativas de suicídio estão relacionadas a déficits no funcionamento executivo. Nessa pesquisa, Keilp et al. (2001) verificaram que pacientes deprimidos com déficit cognitivo tentam em maior frequência o suicídio de alta letalidade quando comparados a outros pacientes depressivos que não apresentaram dificuldades na área cognitiva. Foi observado que os pacientes suicidas de alta letalidade tinham atenção, memória e capacidade de resolução de problemas prejudicados. Outro estudo, desenvolvido por Westheide et al. (2008), corrobora esses achados, constatando que os pacientes depressivos com ideação suicida apresentam déficits no funcionamento executivo das funções cognitivas, o que estaria relacionado principalmente aos comportamentos impulsivos e à dificuldade na resolução de problemas.

Apesar de evidências que apontam alterações neurológicas a partir de estudos de neuroimagem e de avaliações neuropsicológicas, o TPB não é considerado, ainda, uma desordem neurocognitiva definida. No entanto, autores como Monarch et al. (2004), Le Gris e Van Reekum (2006) afirmam que há uma clara falha na literatura acerca de estudos que examinem adequadamente os aspectos cognitivos desses pacientes. Segundo esses mesmos autores, há estudos demonstrando que os pacientes com TPB apresentam habilidades cognitivas prejudicadas. Corroborando esses achados, Monarch et al. (2004) realizaram uma bateria neuropsicológica em doze mulheres portadoras de TPB comparando-as com um grupo controle de mulheres sem patologias mentais. Os resultados indicaram que as 
pacientes com TPB apresentaram prejuízos em sete das nove áreas cognitivas avaliadas, especialmente na atenção, vigilância, memória verbal e capacidade de aprendizado quando comparadas com o grupo controle. Os autores sugerem que seja aplicada uma bateria de testes de inteligência completa nos pacientes com TPB, para esclarecer seu funcionamento cognitivo e melhorar as possibilidades de tratamento.

Outro estudo conduzido por Arza et al. (2009) demonstra que as alterações cognitivas desempenham um papel muito importante nos pacientes com diagnóstico de TPB. Foram avaliados 26 pacientes (14 mulheres e 12 homens) que, comparados a um grupo controle saudável, apresentaram déficits nas funções executivas e em testes neuropsicológicos complexos. Os prejuízos mais evidentes foram na memória imediata, memória de trabalho, atenção, fluência verbal, controle do impulso, flexibilidade cognitiva, abstração e planejamento. As conclusões deste estudo indicam que pacientes com TPB apresentam prejuízos em funções neurocognitivas, apontando ainda que seriam necessários mais estudos para aprofundar o conhecimento dessas alterações.

Em um dos mais importantes artigos de revisão sobre o TPB e comportamento suicida, Le Gris e Van Reekum (2006) consultaram diversas bases de dados buscando artigos a partir do ano de 1985. Foram encontrados 29 artigos discutindo aspectos neuropsicológicos do TPB e sete artigos envolvendo a temática do suicídio. Dos estudos encontrados, 83\% indicavam prejuízos cognitivos em pacientes com TPB, especialmente em funções executivas, tais como tomada de decisão, memória de trabalho e prejuízos na organização visoespacial. Essas dificuldades parecem ser agravadas em pacientes que tentaram suicídio, nos quais os prejuízos cognitivos parecem ainda mais acentuados. Essa revisão da literatura sugere que sejam investigados aspectos globais da inteligência, como a avaliação de QI total, além de outros domínios cognitivos, com o objetivo de identificar com mais clareza essas particularidades que envolvem a cognição de pacientes com ТPB que tentaram suicídio em comparação com os que nunca tentaram.

Já Judd (2005) constatou que pacientes com TPB apresentam grande instabilidade em seus relacionamentos interpessoais, alto grau de impulsividade, dissociação e paranoia, características já bastante enfatizadas pela literatura. Entretanto, essa autora ressalta que as dimensões cognitivas desses pacientes têm sido pouco estudadas, propondo que os prejuízos neurocognitivos podem ser a chave para a compreensão dos aspectos do TPB. A autora hipotetiza, ainda, que prejuízos cognitivos podem estar associados ao apego inseguro e que o fator cognitivo pode ser responsável por muitos comportamentos patológicos das pessoas que apresentam esse transtorno.

Em relação ao TPB, percebe-se que indivíduos suicidas demonstram maiores dificuldades em relação à regulação emocional e à tomada de decisão, devido a alterações neurológicas e cognitivas referentes a déficits na atenção, na memória e no processamento de informações complexas (Le Gris \& Van Reekum 2006; Sprock, Rader, Kendall, \& Yoder, 2000). Contudo, ainda há carência de estudos nessa área, especialmente estudos brasileiros, para que se possa descrever melhor esse fenômeno a fim de gerar subsídios para se repensar intervenções e tratamentos clínicos cada vez mais eficazes para essa grave condição diagnóstica.

\section{Considerações finais}

O TPB é uma patologia de personalidade que necessita ainda de investigação em várias áreas e, apesar de a instabilidade emocional ser sua característica central, muitos outros aspectos ainda permanecem obscuros e pouco explorados em pesquisas. A literatura atual aponta para uma estreita relação entre TPB e déficits cognitivos, sendo esses últimos fundamentais para a compreensão e tratamento desse grave e complexo transtorno que inclui automutilações e tentativas de suicídio como manifestações clínicas frequentes.

Vários domínios cognitivos parecem estar afetados em indivíduos que apresentam o diagnóstico de TPB, especialmente no que se refere às funções executivas, como atenção, concentração, memória de trabalho, tomada de decisões e controle dos impulsos. Em pacientes com TPB com histórico de tentativas de suicídio, os déficits nas funções executivas parecem ser ainda mais acentuados, gerando riscos elevados tanto para a consumação do suicídio quanto para o aumento do número de tentativas cujas consequências são extremamente danosas tanto para o paciente quanto para a família e a rede social da qual o paciente faz parte. 
A avaliação de aspectos cognitivos, nível de impulsividade e ideação suicida de pacientes com TPB deve ser feita criteriosamente devido aos riscos inerentes a essa grave psicopatologia de personalidade. A relevância em se avaliar os aspectos cognitivos, a impulsividade e o risco de suicídio em pacientes com TPB, reside na possibilidade de gerar subsídios para melhorar as formas de intervenção nesse transtorno e, especialmente, evitar o comportamento suicida e o grave risco de morte em que ele implica.

\section{Referências bibliográficas}

American Psychiatric Association. (2002). Manual diagnóstico e estatístico de transtornos mentais (DSM-IVTR). (4a ed). Porto Alegre: Artmed.

Anestis, M. D., Selby, E. A., \& Joiner, T. E. (2007). The role of urgency in maladaptive behaviors. Behavior Research and Therapy, 45, 3018-3029.

Akiskal, H. S., Downs, J., Jordan, P, Watson, S., Daugherty, D., \& Pruitt, D. B. (1985). Affective disorders in referred children and younger siblings of maniacdepressives: mode of onset and prospective course. Archives of General Psychiatry, 42, 996-1003.

Arza, R., Diaz-Marsá, M., López-Micó, C., Pablo, N. F., LópezIbor, J. L., \& Carrasco, J. L. (2009). Neuropsychological dysfunctions in personality borderline disorder: detection strategies. Actas Españolas de Psiquiatría, 37(4), 185-190.

Bazanis, E., Rogers, R. D., Dowson, J. H., Taylor, P, Meux, C., Staley, C., \& Sahakian, B. J. (2002). Neurocognitive deficits in decision-making and planning of patients with DSM-III-R borderline personality disorder. Psychological Medicine, 32(8), 1395-1405.

Brezo, J., Paris, J., \& Turecki, G. (2006). Personality traits as correlates of suicidal ideation, suicide attempts, and suicide completions: a systematic review. Acta Psychiatrica Scandinavia, 113, 180-206.

Black, D. W., Blum N., Pfohl, B., \& Hale, B. (2004). Suicidal behavior in borderline personality disorder: Prevalence, risks factors, prediction and prevention. Journal of Personal Disorder, 18(1), 226- 239.

Bowlby, J. (1969/1973). Attachment and loss: Vol. 1. Attachment. New York: Basic Books.
Brodsky, B. S., Malone, K. M., Ellis, S. P, Dulit, R. A., \& Mann, J. J. (2006). Characteristics of borderline personality disorder associated with suicidal behavior. American Journal of Psychiatry, 154, 1715-1719.

Brooke, S., \& Horn, N. (2010). The meaning of selfinjury and overdosing amongst women fulfilling the diagnostic criteria for borderline personality disorder. Psychology and Psychotherapy, 83(2), 113-115.

Domes, G., Winter, B., Schneel, K., Vohs, K., Fast, K., \& Herpertz, S. C. (2006). The influence of emotions on inhibitory functioning in borderline personality disorder. Psychological Medicine, 36, 1163-1172.

Freeman, J. B., \& Beck, G. (2000). Cognitive interference for trauma cues in sexually abused adolescent girls with posttraumatic stress disorder. Journal of Clinical and Child Psychology, 29, 245-56.

Gabbard, G. O. (1998). Psiquiatria psicodinâmica: Baseado no DSM-IV. Porto Alegre: Artmed.

Gabbard, G. O., \& Wilkinson, S. M. (1994). Management of countertransference with borderline patients. Washington, DC: American Psychiatric Press.

Gunderson, J. G., \& Sabbo, A. N. (1993). The phenomenological and conceptual interface between borderline personality disorder and PTSD. American Journal of Psychiatry, 150, 19-27.

Gvion, Y., \& Apter, A. (2011). Aggression, impulsivity, and suicide behavior: A review of the literature. Archives of Suicide Research, 15(2), 93-112.

Herman, J. L., Perry, J. C., \& Van der Kolk, B. A. (1989). Childhood trauma in borderline personality disorder. The American Journal of Psychiatry, 146(4), 490-495.

Johnson, J. G., Cohen, P, Smailes, E. M., Skodol, A. E., Brown, J., \& Oldham, J. M. (2001). Childhood verbal abuse and risk for personality disorders during adolescence and early adulthood. Comprehensive Psychiatry, 42(1), 16-23.

Joyce, P. R., Light, K. J., Rowe, S. L., Cloninger, C. R., \& Kennedy, M. A. (2010). Self-mutilation and suicide attempts: Relationships to bipolar disorder, borderline personality disorder, temperament and character. Australian and New Zealand Journal of Psychiatry, 44(3), 250-257. 
Judd, P. H. (2005). Neurocognitive impairment as a moderator in the development of borderline personality disorder. Development and Psychopathology, 17, 1173-1196.

Kaplan, H., \& Sadock, B. (2008). Compêndio de Psiquiatria: ciências do comportamento e psiquiatria clínica. Porto Alegre: Artes Médicas.

Keilp, J. G., Sackeim, H. A., Brodsky, B. S., Oquendo, M. A., Malone, K. M., \& Mann, J. (2001). Neuropsychological dysfunction in depressed suicide attempters. American Journal of Psychiatry, $158,735-741$.

Kernberg, 0. (1998). Psicoterapia psicodinâmica de paciente borderline. Porto Alegre: Artes Médicas.

Koenigsberg, H. W. (2002). Characterizing affective instability in borderline personality disorder. American Journal of Psychiatry, 159(5), 784-788.

Le Gris, J., \& Van Reekum, R. (2006). The neuropsychological correlates of borderline personality disorder and suicidal behavior. The Canadian Journal of Psychiatry, 51, 131-142.

Levy, K. N., Meehan, K. B., Kelly, K. M., Reynoso, J. S., Weber, M., Clarkin, J. F., \& Kernberg, O. F. (2006). Change in attachment patterns and reflective function in a randomized control trial of transference-focused psychoteraphy for borderline personality disorder. Journal of Consulting and Clinical Psychology, 74(6), 1027-1040.

Lieb, K., Zanarini, M. C., Schmahl, C., Linehan, M. M., \& Bohus, M. (2004). Borderline Personality Disorder. The Lancet, 364, 453-461.

Linehan, M. (2010). Terapia cognitivo-comportamental para transtorno de personalidade borderline. Porto Alegre: Artmed.

Links, P. S., Heisel, M. J., \& Garland, A. (2003). Affective Instability in personality disorders. American Journal of Psychiatry, 160, 394-395.

Lyons-Ruth, K., Jacobvitz, D., Cassidy, J., \& Shaver, P R. (1999). Attachment disorganization: Unresolved loss, relational violence, and lapses in behavioral and attentional strategies. In J. Cassidy, \& P R. Shaver (Eds.), Handbook of attachment: Theory, research, and clinical applications (pp 520-554). New York: Guilford Press.
Maloney, E., Degenhardt, L., Darke, S., \& Nelson, E. C. (2009) Impulsivity and borderline personality disorder as risk factors for suicidal attempts among opioid-dependents individuals. Psychiatry Research, 16(1), 16-21.

Mann, J. (2003). Neurobiology of suicidal behavior. Nature Reviews Neuroscience, 4, 819-828.

McLean, L. M., \& Gallop, R. (2003). Implications of childhood sexual abuse for adult borderline personality disorder and complex posttraumatic stress disorder. The American Journal of Psychiatry, 160(2), 369-371.

Monarch, E. S., Saykin, A. J., \& Flashman, L. A. (2004). Neuropsychological impairment in borderline personality disorder. Psychiatric Clinics of North America, $27,67-82$.

New, A. S., Goodman, M., Triebwasser, J., \& Siever, L. J. (2008). Recent advances in the biological study of personality disorders. Psychiatry Clinics and North America, 31, 441-61.

Nigg, J. T., Lohr, N. E., Western, D., Gold, L. J., \& Silk, K. R. (1992). Malevolent object representations in borderline personality disorder and major depression. Journal of Abnormal Psychology, 101(1), 61-67.

O’Connor, R. C., \& Noyce, R. (2008). Personality and cognitive processes: Self-criticism and different types of rumination as predictors of suicidal ideation. Behavior Research and Therapy, 46, 392-401.

O’Donohue, W., \& Cucciare, M. A. (2007). Identifying and treating psychological factors in medical settings: the example of borderline personality disorder. International Journal of Clinical and Health Psychology, 7(3), 807-821.

O’Leary, K. M., Brouwers, P, Gardner, D. L., \& Cowdry, R. W. (1991). Neuropsychological testing of patients with borderline personality disorder. The American Journal of Psychiatry, 148(1), 106-111.

Organização Mundial da Saúde. (2001). Relatório sobre a saúde no mundo 2001. Genebra, Suíça: Autor.

Organização Mundial da Saúde. (1994). CID-10: classificação internacional de doenças. São Paulo: EDUSP.

Paris, J. (2005). The development of impulsivity and suicidality in borderline personality disorder. Development Psychopathology, 17, 1091-1104. 
Poletti, M. (2009). Neuro cognitive functioning in borderline personality disorder. Rivista di Psichiatria, 44(6), 374-383.

Ruocco, A. C. (2005). The neuropsychology of borderline personality disorder: A meta-analysis and review. Psychiatry Research, 137(3), 191-202.

Romaro, R. (2002). O sentimento de exclusão social em personalidade borderline e o manejo da contratransferência. Revista Mudanças, 10(1), 62-71.

Salzman, J. P., Salzman, C., Wolfson, A. N., Albanese, M., Looper, J., Ostacher, M., \& Miyawaki, E. (1993). Association between borderline personality structure and history of childhood abuse in adult volunteers. Comprehensive Psychiatry, 34(4), 254-257.

Soler, J., Pascual, J. C., Tiana, T., Cebrià, A., I., Barrachina, J., Campins, J., \& Pérez, V. (2009). Dialectical behaviour therapy skills training compared to standard group therapy in borderline personality disorder: a 3-month randomized controlled clinical trial. Behaviour Research and Therapy, 47(5), 353-358.
Soloff, P. H., Pruitt, P., Sharma, M., Radwan, J., White, R., \& Diwadkar, V. A. (2012). Structural brain abnormalities and suicidal behavior in borderline personality disorder. Journal of Psychiatry Research, 46(4), 516-525.

Sprock, J., Rader, T. J., Kendall, J. P., \& Yoder, C. Y. (2000). Neuropsychological functioning in patients with borderline personality disorder. Journal Clinical Psychology, 56, 587-600.

Westheide, J., Quednow, B. B., Kuhn, K. U., Hoppe, C., Cooper-Mahkorn, D., Hawellek, B., \& Wagner, M. (2008). Executive performance of depressed suicide attempters: The role of suicidal ideation. European Archives of Psychiatry and Clinical Neuroscience, 258(7), 414-421.

Zanarini, M. C., Frankenburg, F. R., \& Vujanovic, A. A. (2002). Inter-rater and test-retest reability of the revised diagnostic interview for borderlines. Journal of Personality Disorders, 16(3), 270-276.

Zelkowitz, P., Paris, J., Guzder, J., \& Feldman, R. (2001). Diatheses and stressors in borderline pathology of childhood: The role of neuropsychological risk and trauma. Journal of the American Academy of Child \& Adolescent Psychiatry, 40(1), 100-105. 